\title{
Tools to Engineer the Genome
}

\section{BioTechniques Focus}

Advances in genetic engineering techniques and methods have led to breakthroughs in scientific disciplines such as synthetic biology. For this special BioTechniques Focus section, the editors have selected two articles describing methods and applications for engineering large sections of DNA. First, in a special news feature, editor-in-chief Nathan Blow details the history of synthetic gene circuits, and speaks with several researchers who are engineering novel synthetic circuits in cells for applications ranging from logic computation to metabolite production. In our second article, a team of researchers from Malaysia describe a new methodology for directed evolution of nucleotide libraries that bypasses many limitations found in traditional random mutagenesis and DNA shuffling approaches. We hope these articles provide all readers with a better sense of the latest advances in genome engineering technology, as well as a clearer understanding of how these tools are being used in modern synthetic biology.

SEM image of Mycoplasma mycoides JCVI syn 1.0, courtesy of Thomas Deerinck and Mark Ellisman, NCMIR and John Glass and J. Craig Venter, JCVI.
INTRODUCTION

349 Genome engineering: methods and applications The Editors, BioTechniques doi $10.2144 / 000113968$

\section{FEATURES}

\section{Hot-wiring cells}

Nathan Blow, Ph.D.

doi 10.2144/000113965

357 Directed evolution of nucleotide-based libraries using lambda exonuclease

Bee Nar Lim, Yee Siew Choong, Asma Ismail, Jörn Glökler, Zoltán Konthur and Theam Soon Lim doi 10.2144/000113964 